\title{
El cuento multimodal como estrategia didáctica para el aprendizaje de la lectura
}

Gladis Cecilia Rojas Camargo* Magda Liced Prieto Martínez ${ }^{* *}$

Artículo de reflexión

Fecha de Recepción: 27 febrero 2018.

Fecha de Aprobación: 30 junio 2018.

\section{Resumen}

Este artículo presenta los resultados de la investigación: "La lectura inicial, reto en la escuela actual", cuyo propósito es motivar la lectura en estudiantes de grado primero, mediante una estrategia pedagógica-didáctica, donde se aprenda no a leer por leer, sino leer para aprender. Esta se fundamenta, entre otras, en teorías de: Acevedo (2014), con el funcionamiento del cerebro de los niños; Freinet (1981), con el método natural de lectura; Ana María Maqueo, con el enfoque comunicativo en la enseñanza del español; Hurtado (2003), procesos psicolingüísticos en la lectura; Rincón (1999, con el aprendizaje y el error constructivo. La investigación se desarrolla con 50 estudiantes de grado primero del Colegio Técnico Municipal
"Simón Bolívar"de las sedes Central y el Mirto, de Duitama. Se enmarca en el paradigma cualitativo, enfoque socio-crítico e Investigación Acción Educativa. La metodología tiene como fundamento el cuento multimodal. Las técnicas e instrumentos que se utilizaron fueron: la observación, la clase entrevista, el diario de campo y talleres didácticos. El desarrollo de la investigación permitió concluir que los estudiantes se ven motivados a leer cuando se les presenta textos con diferentes estructuras $y$, en especial, los que se proyectan utilizando herramientas TIC.

Palabras Clave: alfabetización, promoción de la lectura, motivación, cuento, talleres didácticos.

\section{* Colegio Técnico \\ Municipal "Simón Bolívar", \\ Duitama, sede el Mirto glacero69@yahoo.es. $* *$ Colegio Técnico Municipal "Simón Bolívar", Duitama, sede Central magshanon@gmail.com}

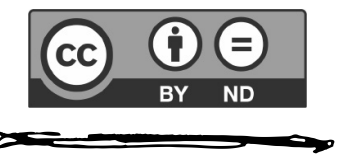




\section{Introducción}

Uno de los propósitos básicos de la educación, particularmente, de la básica primaria, es el de enseñar a leer en forma comprensiva, reflexiva y critica, a los estudiantes, dada la importancia de esta habilidad en el crecimiento integral, social, cultural y académico del ser humano. En consecuencia, uno de los retos de la escuela, es el de ofrecer espacios propicios para lograrlo.

Producto de la observación de prácticas pedagógicas se infiere la oportunidad de mejoramiento en la fundamentación teórica del maestro en relación con los procesos lecto-escriturales. Tal debilidad, en ocasiones, conlleva la utilización de metodologías no pertinentes. En este contexto, la lectura se considera como una simple mecanización (deletreo, silabeo), que conlleva al estudiante a una lectura mecánica, sin sentido, lo que hace que pierda la motivación y el interés, componentes fundamentales para el proceso de desarrollo cognitivo. Esta situación se refleja, en el caso particular, en los bajos resultados en el área de Lenguaje, que arroja el Índice Sintético de Calidad Educativa, ISCE, 2015 y 2016, de la Institución Educativa Técnico Municipal "Simón Bolívar", de Duitama.

En este ámbito, se piensa la propuesta pedagógico-didáctica "El cuento multimodal como estrategia para la enseñanza-aprendizaje de la lectura", cuyo horizonte es dar respuesta a la pregunta ¿Cómo favorecer la enseñanza-aprendizaje de la lectura, en los estudiantes de grado primero de las Sedes "Central" y "Mirto", del Colegio Técnico Municipal "Simón Bolívar", de Duitama?. La propuesta se desarrolla en el marco del enfoque socio-crítico y de la Investigación Acción Educativa, previo diagnóstico, se diseñó y desarrolló una estrategia que utiliza los talleres didácticos, los cuales tienen como elemento motivador el cuento multimodal. Lo anterior, para facilitar el proceso de apropiación de la lectura.

\section{Planteamiento del Problema de investigación}

En el desarrollo del proceso de investigación educativa es importante abordar el tema de la enseñanzaaprendizaje de la lectura; en palabras de Mendoza (2006), "la lectura es hoy, no sólo preocupación de muchos docentes, sino también un problema para miles de estudiantes" (p. 219), en los cuales se percibe dificultades en su adquisición; lo cual se convierte en una barrera que les impide acceder a una mejor formación integral, evidenciado en el desarrollo de las actividades escolares y sociales; por ello, se implementa una estrategia para mejorar el proceso de enseñanzaaprendizaje de la competencia lectora, que permita a los discentes darle sentido y promover la necesidad de leer como base para interactuar con los demás en el presente y el futuro.

Teniendo en cuenta las características de los estudiantes que ingresan a grado primero, se evidencia dificultades motivacionales y sociales en ellos, que no son tenidas en cuenta por el maestro 
en el proceso de enseñanza-aprendizaje, lo cual incide, de manera desfavorable en el desarrollo del proceso lector, situación que le dificulta al niño tener un buen desempeño en su vida escolar y social.

Por otra parte, en el desarrollo metodológico que se viene adelantando en la institución, se observa que la forma como se orienta el proceso lector no se fundamentan en el pensamiento ni en el lenguaje, sino que se considera como una simple mecanización (deletreo, silabeo), utilizando el método sintético que conlleva al estudiante a una lectura mecánica, sin sentido, lo que le hace perder la motivación y el interés, componentes fundamentales para el proceso de desarrollo cognitivo. A la vez, y como lo manifiesta Carminatti (2012) los docentes "en la vida cotidiana nos enfrentamos a situaciones que causan efectos nocivos en la persona: falta de atención a las emociones, mala alimentación, estrés, violencia y deficiencias en la educación” (p. 34), y nuestra institución no es ajena a ello.

Prueba de lo mencionado anteriormente son los resultados que arrojan el Índice Sintético de Calidad Educativa (ISCE) 2016 y 2017, de la Institución, donde se evidencia las dificultades que presentan los estudiantes en cuanto a la lectura, comprensión e interpretación de textos. Esta situación también se observa en su desempeño dentro del aula, con lo cual se ratifica la necesidad de mejorar el proceso de apropiación y desarrollo de la habilidad lectora, para ampliar el léxico, optimizar el desempeño académico y social de los niños. Pues, una de las competencias básicas que debe tener un estudiante es la de aprender a aprender, en donde la competencia lectora juega un papel importante. Para ello, se debe desarrollar actividades que promuevan el goce y el disfrute al leer.

Una situación que acrecienta el problema es la de que el Ministerio de Educación Nacional, (MEN), promueve políticas poco acertadas para la ubicación y asignación de maestros, lo hace sin respetar el área disciplinar del pregrado realizado por el docente, en estas circunstancias hay profesionales que no están preparados para desempeñarse en primaria y menos para enseñar a leer a los estudiantes. Respecto de esta problemática, la institución no es ajena a ello, ya que, se encuentran docentes que a pesar de poseer las características necesarias, la vocación, el amor por su profesión, infortunadamente, carecen de la preparación y perfil para desenvolverse como guía en el proceso de iniciación a la lectura; esto conlleva replantear las políticas y directrices del MEN, respecto del nombramiento de maestros, así, como la asignación del nivel educativo en el cual se deben desempeñar.

\section{Antecedentes}

En el desarrollo de la investigación se consultó entre otros las siguientes investigaciones:

\section{En el ámbito internacional.}

Bravo (2002), quien en su propuesta muestra cómo los procesos cognitivos que componen la conciencia fonológica

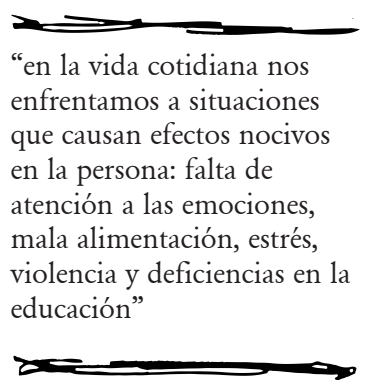


Navarro (2015), en esta investigación se hace un análisis de la pedagogía tradicional utilizada en el desarrollo de la habilidad lectora, la cual sirve de base para evaluar la manera con la cual se viene enseñando a leer y así promover nuevas formas de realizar este proceso. evidencian una mayor variabilidad común con el aprendizaje de la lectura, como determinante para su éxito o fracaso para aprender a leer. El autor, igualmente, pone de manifiesto que el niño debe tener unas condiciones para aprender a leer, y si el profesor tiene en cuenta componentes como el fonológico y la parte oral conseguirá que el niño vaya descubriendo fácilmente el gusto o agrado por la lectura; además, al conocer el nivel de desarrollo en que se encuentra el niño se puede aplicar, crear o proponer estrategias que busquen que él interactúe con esos dos componentes.

Otros aportes importantes que ofrece esta propuesta son: en la parte pedagógica, la necesidad de hacer un diagnóstico de los niveles de conocimiento o proceso cognitivo del niño; y en la parte psicológica, conocer la etapa de desarrollo cognitivo en la que se encuentra y a partir de allí comenzar a aplicar estrategias que le hagan más agradable iniciar su proceso lector.

Lara (2008), en su trabajo presenta estrategias para la enseñanza aprendizaje de la lecto-escritura en el primer grado de educación primaria, dirigidas al desarrollo de los procesos básicos para la adquisición del código de la lengua, tanto oral como escrita; estas estrategias, según el autor, pueden ser empleadas en cualquier aula de primer grado, ofreciendo orientaciones fundamentales en el desarrollo de esta aventura investigativa.

Lorenzo (2012) en su investigación, explica los diferentes métodos para la enseñanza de la lectura, la historia de la evolución alfabética y la conciencia fonológica; las cuales intervienen en el desarrollo de la iniciación a la lectura. Propone la utilización de diversos métodos para la alfabetización y promoción de la lectura, ejes fundamentales de esta investigación.

Navarro (2015), en esta investigación se hace un análisis de la pedagogía tradicional utilizada en el desarrollo de la habilidad lectora, la cual sirve de base para evaluar la manera con la cual se viene enseñando a leer y así promover nuevas formas de realizar este proceso. A la vez muestra alternativas para fomentar las habilidades orales, el trabajo en grupo creando ambientes de aprendizaje colaborativo el cual es uno de los propósitos de esta propuesta.

\section{En el ámbito nacional.}

Prieto (2015), esta investigación tiene como propósito lograr que los niños inicien el hábito lector, mediante actividades lúdico pedagógicas dirigidas a leer y explicar lo leído, priorizando en las estrategias de acuerdo al momento de la lectura: antes, durante y después, lo cual es de gran importancia para promover y animar la comprensión lectora, así mismo un adecuado comportamiento lector que va a otorgar a la persona una dimensión participativa, constructiva, creadora en su entorno natural y social. Por último, Jimenez \& Gordo (2014) plantean como el cuento la potencia pedagógica que tiene el cuento como facilitador del pensamiento en los niños. 


\section{Fundamentación Teórica Conceptual}

Con respecto a los componentes conceptuales que integran la investigación ya referida, es primordial conocer cómo trabaja el cerebro del niño, para lo cual, se retoman las concepciones de Acevedo (2014) cuando afirma que "los padres y profesores son quienes cambian y esculpen los cerebros de los infantes" (p. 9), por lo tanto, es fundamental que se conozca su funcionamiento, para determinar la edad más favorable para el desarrollo del proceso lector $\mathrm{y}$, así, tener una visión más clara para incentivar el aprendizaje de la lectura.

Así mismo, la adquisición del lenguaje es un proceso natural que todo ser humano experimenta desde su nacimiento al tener impulsos constantes de crecimiento y perfeccionamiento, mediante los cuales el niño manifiesta necesidades fisiológicas funcionales que le permiten acomodarse a las exigencias del entorno, imitando y corrigiendo los defectos hechos del lenguaje, para pasar del tanteo experimental a la adquisición de habilidades comunicativas, las cuales deben ser guiadas por una persona versada en el tema, de manera que se pueda oportunamente ir mejorando el proceso de aprendizaje (Freinet, 1981), en particular de la lectura.

Cabe recordar que, según Acevedo (2014), "el cerebro siempre busca la novedad, en otras palabras, está genéticamente preparado para aprender algo nuevo" (p.10). Para ello posee zonas especializadas que están listas para ser utilizadas solamente una vez en la vida, por ello, es necesario estimular el cerebro para aprovechar estos "momentos sensibles" que influyen en el proceso de aprendizaje de la lectura, los cuales se dan entre los 6 y 8 años, periodo en el cual el cerebro está listo para darle significado y sentido a los grafemas y fonemas, como elementos importantes en el lenguaje. Lo que se aprende en estos momentos se evoca con mayor facilidad, por lo tanto, es importante que todos los docentes estén informados acerca de su funcionamiento, para que, de esta manera, puedan tener una mejor comprensión y entiendan cómo influye en la adquisición del proceso lector y así puedan ayudar al estudiante a afrontar esta etapa, de una forma natural, incorporando la lectura tanto en su vida cotidiana.

Cabe anotar que el lenguaje es esencial no solo para nuestra supervivencia física y social, sino también para compartir el saber colectivo que se construye en familia, comunidad y sociedad. La verdad es que los niños llegan a la escuela ricos en experiencias de lecto-escritura y también ya han aprendido a darle significado a lo impreso. Gradualmente aprenden que lo impreso tiene sentido; qué clase de significado tiene, cómo lo adquiere, es decir, llegan a controlar el sistema del lenguaje (Goodman, 2003).

Además, en el funcionamiento biológico del sujeto lector influyen todos los sentidos, es decir no solo se leen códigos y signos, por el contrario, se pueden 
Ha sido entendido como el instrumento de mayor excelencia y flexibilidad para la comunicación, como el sistema simbólico más elaborado, como el basamento obligado sobre el que se edifica la propia identidad, puesto que el lenguaje da cause a la autoexpresión. Y es la pieza capital que nos permite la comprensión y la ordenación de lo que somos y de lo que nos rodea. leer gestos faciales, notas musicales, mapas, pinturas, sonidos, texturas; al leer el estudiante recuerda, establece relaciones, diferencias y da coherencia a la nueva información percibida, para llevarla a construir conocimientos y desarrollar habilidades naturales, verdaderamente significativas. En este sentido, la propuesta se centra no solo en leer por leer, sino leer para aprender, utilizando diferentes herramientas que le permitan al estudiante interactuar con su ámbito social.

Por otra parte, el lenguaje, además de poseer una función cognitiva y poética tiene una función comunicativa, donde los sujetos interactúan entre sí en forma oral y escrita; con lo cual desarrollan su competencia comunicativa (CC), la cual, según Maqueo (2005) "consiste en entablar y sostener una relación interpersonal valiéndose de una serie de comportamientos verbales y no verbales para permitir la comunicación con diferentes personas y en diferentes situaciones" (p. 164), donde el lenguaje se convierte en facilitador para desarrollar la CC, que le permite al estudiante interactuar, desarrollar habilidades sociales que lo llevan, entre otros aspectos, a poseer un vocabulario enriquecido, lo cual posibilita una mejor comprensión de los textos que se le presentan y, de esta manera, tener un mejor desempeño escolar y social. Cabe recordar que la comunicación humana de calidad requiere, igualmente, según Maqueo (2005), de "conocimientos lingüísticos, sociales y culturales, para que se lleve a cabo una comunicación adecuada en situaciones reales" (p. 166). Tal competencia es la base sobre la que se asienta el enfoque comunicativo.
Así mismo, se debe tener claridad acerca de lo que es el lenguaje y para ello se retoma a Tusón (1989) citado por Maqueo (2005), quien expone que este

Ha sido entendido como el instrumento de mayor excelencia y flexibilidad para la comunicación, como el sistema simbólico más elaborado, como el basamento obligado sobre el que se edifica la propia identidad, puesto que el lenguaje da cause a la autoexpresión (Colella, 2016). Y es la pieza capital que nos permite la comprensión y la ordenación de lo que somos y de lo que nos rodea. (p. 171)

Lo anterior permite considerar el lenguaje como eje en el desarrollo de la lectura, primordial para la expresión oral, la comprensión lectora; habilidades básicas para el inicio de la escolaridad.

Es por ello que, en la iniciación del niño en la lectura, se debe tener en cuenta, según la teoría piagetiana, que "él es ante todo un sujeto activo, capaz de comprender el mundo que lo rodea y de resolver los problemas que en él se le presenten, es decir, es un sujeto que permanentemente está construyendo conocimiento" (citado en Hurtado et al, 2003, p. 21), lo cual hace que el niño le otorgue significado a lo que aprende en la escuela y a la información que obtiene de su entorno; por esto, se le deben proporcionar textos con diferentes temáticas, ricos en léxico y con situaciones que sean relevantes para él.

En este sentido, es indispensable tener en cuenta las dos habilidades en la 
iniciación a la lectura: hablar y escuchar. Capacidades que se deben desarrollar en los niños para recordar las palabras que necesita para identificar o describir lo que les interesa. Por ello, es útil crear situaciones en las que se expongan a nuevo vocabulario y en las cuales haya un tema de interés. Actividades como lectura de cuentos, canciones, retahílas, poemas, historias, entre otros (MEN, 2016), ayudan a mejorar la comprensión lectora y la expresión oral. Además, para lograr un desarrollo eficiente en la lectura, se deben tener en cuenta los siguientes elementos: conocer bien el idioma en el que se está aprendiendo a leer, poseer buenas habilidades auditivas y, por último, tener buenas habilidades visuales $\mathrm{y}$ viso-motoras.

De igual manera, "Leer en la educación inicial, se entiende, en el sentido amplio de desciframiento vital de posibilidades interpretativas $y$ de exploración de mundos simbólicos, esto no implica enseñar letras descontextualizadas, hacer planas o alfabetizar prematuramente" (MEN, 2016, p. 11). Por el contrario, la lectura, según el MEN (1996) "es un proceso de construcción de significados a partir de la interacción entre el texto, el contexto y el lector" (p. 72), en la que la percepción del lector es importante para identificar las unidades lingüísticas, visualizar, evocar conocimientos a través de la memoria y activar ciertos esquemas para comprender el texto. Para ello, se considera que el trabajo en equipo facilita la confrontación de saberes y genera mayor comprensión de la realidad. Al respecto Teberosky
(1982) plantea que "esta interacción constituye una fuente de conflicto, puesto que los niños utilizan sus propias hipótesis para asimilar la información del medio, y las ponen a prueba al confrontarlas con las hipótesis de otros, no siempre idénticas a las suyas" (Citado en Hurtado, et al, 2003, p. 15).

Así mismo, como lo explica Alliende, (1982), "la capacidad que tiene el sujeto para asimilar conceptos, acomodar, modificar la información y resolver problemas, comprendiendo la nueva información, mejorando su parte emocional, y la competencia lingüística, entendida como el conjunto de reglas gramaticales" (Citado en MEN, 1996 p.75), deben ser tenidas en cuenta por el docente al momento de iniciar el proceso de lectura, ya que se requiere que el estudiante tenga un cerebro que le permita ser capaz de leer, recordar y activar estos recuerdos cada vez que percibe signos que ha conocido previamente, impactando el cerebro, alterando las configuraciones neuronales para representarlas en la mente; en el caso de los signos, esta representación debe incluir el concepto, la palabra y la asociación entre ambos. Solo así será posible que esa configuración se genere ante la percepción de algo similar a lo visto originalmente, iniciando su proceso de alfabetización, y desarrolle el sistema de comunicación que denominamos lenguaje (Parodi, 2010).

En este sentido, Rincón, Bustamante \& Pérez, (1999) afirman que el aprendizaje es la confrontación de 
nociones y teorías con acciones e ideas que producen cambios conceptuales, y toman el "error constructivo" como la principal herramienta y requisito para la construcción de conocimiento; como lo han demostrado Ferreiro \& Teberosky (1979), el aprendizaje comienza extraescolarmente, y es a partir de las diversas interacciones sociales con pasacalles, grafitis, logotipos, periódicos y revistas, entre otros, como los niños comienzan a construir la lengua escrita. Por lo mismo, es necesario promover experiencias de lectura que permitan la confrontación de los diferentes niveles de conceptualización de los niños, dinamizando el proceso de desarrollo de la lectura, que, según Hurtado at al (2003), "realimenta las diversas producciones lingüísticas de los niños, permitiéndoles tomar conciencia de cómo están leyendo, generando un proceso de autocorrección $y$ transformación lingüística y cognitiva" (p. 15).

Otro aspecto importante es la alfabetización inicial o lectura emergente, la cual, consiste en desarrollar estrategias, habilidades y conocimientos que favorezcan el aprendizaje de la lectura y la escritura, y que se desarrollan desde el nacimiento hasta cuando el niño ya lee convencionalmente (MEN, 2016). Por lo anterior, es primordial tener en cuenta la motivación, como el motor que permite actuar en el entorno escolar, siendo absolutamente imprescindible fomentarla y educarla. Al respecto, Ian Gilbert (2005) expresa: "No me he encontrado aún con ningún niño que no esté motivado, sino que a veces ocurre simplemente que no están motivados para hacer lo que deseamos que hagan y cuando queremos que lo hagan" (Citado en MEN 2016, p.11). Los profesores pueden utilizar las estrategias educativas adecuadas para enseñar y motivar a los alumnos para responsabilizarse de su aprendizaje, haciendo que quieran y que hagan las actividades escolares en forma dinámica, activa y participativa, para que así, sea el niño el generador de su aprendizaje (Guillen, 2014).

Lo anterior lleva a tener en cuenta la Teoría de la Modificabilidad Cognitiva propuesta por Feuerstein quien plantea que "el ser humano es un organismo abierto al cambio, con una inteligencia dinámica, flexible y receptora a la intervención positiva de otro ser humano.” (Feuerstein, 2011) de ahí la importancia de que el docente tome en cuenta los factores que inciden en la Modificabilidad Cognitiva y promueva la adaptación de los niños al ambiente escolar para crear uno propicio, donde se utilice diferentes actividades innovadoras y motivadoras, se fomente la imaginación, la creatividad de los estudiantes y, así, lograr elementos que favorezcan la habilidad lectora.

Teniendo en cuenta los anteriores aspectos, se buscó un método que favoreciera el proceso de enseñanzaaprendizaje de la lectura y que coincidiera con el propósito de la investigación; para ello, se toma el cuento como herramienta de motivación a la lectura, ya que como lo expresa el MEN 2007: 
Al leer en voz alta, se desarrolla la capacidad de atención y el vocabulario; se ayuda a que el niño exprese sus emociones, temores, esperanzas, descubrimientos, pensamientos y secretos. Al compartir estos sentimientos se establece un lazo afectivo entre el maestro y los niños, que favorece el proceso de aprendizaje de la lectura y la escritura. Así mismo, los cuentos también ayudan a orientar conductas y afianzar valores.

En cuanto específicamente al cuento multimodal Kress \& Van Leeuwen (2001), (Citado en Moya \& Pinar, 2007 p. 22) expresan que este "utiliza una ampliagamademodos derepresentación y comunicación de los mensajes, que incluye diagramas, fotografías, videos, diversos formatos de página, colores y diagramaciones específicos que contribuyen en gran medida en la comprensión e interpretación del mensaje transmitido". Tales ventajas se convierten en una fuente de motivación para la lectura.

Con base en los planteamientos anteriores se implementó la propuesta pedagógica que se explica en el apartado siguiente.

\section{Metodología.}

Diseño. La experiencia investigativa se enmarca en el paradigma cualitativo, con un enfoque sociocrítico y se desarrolla a través de la Investigación Acción Educativa dado que esta, pretende construir y formular alternativas de acción, específicamente, mejorar la practica pedagógica.
Muestra. 50 estudiantes cuyas edades oscilan entre 5 y 8 años, de la institución educativa Colegio Técnico Municipal "Simón Bolívar", sedes el "Mirto" y "Central". Los educandos, provienen de los estratos 0, 1 y 2, en cuyos hogares predomina la descomposición familiar por diferentes circunstancias, necesidades afectivas, psicológicas, sociales y económicas. Los padres de familia se desempeña en trabajos informales y algunos son empleados, cuya formación académica no supera la Educación Media; la gran mayoría, apenas terminaron la Primaria. Otra de las características que presenta la muestra es el ser población flotante, lo cual dificulta un seguimiento riguroso de los diferentes procesos educativos.

Es importante resaltar que los procedimientos utilizados en el desarrollo de la investigación así como en la implementación de la propuesta han sido realizados tras obtención de la firma del Consentimiento Informado, por los 50 padres de familia de los estudiantes, objeto de estudio.

Diseño de la investigación. Este es coherente con el establecido por Carr \& Kemmis (1989), para una Investigación Acción desarrollando las fases: planificación, acción, observación y reflexión.

\section{Planificación.}

\section{Etapa Diagnóstica.}

En esta etapa se realizó:

- Un diagnóstico, teniendo en cuenta la revisión documental:
Al leer en voz alta, se desarrolla la capacidad de atención y el vocabulario; se ayuda a que el niño exprese sus emociones, temores, esperanzas, descubrimientos, pensamientos y secretos. 
PEI, mallas curriculares, estándares, DBA, competencias, desempeños, resultados obtenidos en el ISCE y prueba SABER 2015 y 2016, en relación con el área de lenguaje, y el Plan de Mejoramiento de la Institución; así mismo, antecedentes y autores versados en la temática.

- Una autorreflexión y análisis de las practicas pedagógicas realizadas por las autoras de esta investigación

- La aplicación de la Clase entrevista diseñada por Grossi (2010), la cual permite ubicar a cada estudiante en el nivel de psicogénesis en lectura.

- Estructuración de propuesta pedagógica: "El cuento multimodal como estrategia de enseñanzaaprendizaje de la lectura”, la cual consta de siete talleres, los tres primeros, orientados a motivar el gusto por la lectura y, los cuatro últimos, a desarrollar la competencia lectora mediante cuentos multimodales que suscitan a la lectura, teniendo en cuenta los intereses, experiencias vivenciales, situaciones de la vida cotidiana de los estudiantes; para ocasionar en ellos un papel activo que convierta el aprendizaje en un reto, motivación, un espacio educativo significativo para crear y desarrollar nuevas capacidades.

Acción. Implementación de la propuesta, mediante el desarrollo de los talleres:

- Taller 1: "La lectura, entrada a un mundo mágico", con base en el cortometraje, "El mejor cortometraje del mundo para motivar a la lectura”. Propósito: promover el gusto y el interés por la lectura.

- Taller 2: "Explorando un mundo de fantasía”. Propósito: Familiarizar al niño con los libros, mediante la visita a la Biblioteca de Comfaboy, lugar que proporciona diversidad de actividades encaminadas a promover el gusto por la lectura.

- Taller 3: "Construyendo un lugar mágico". Propósito: promover el contacto del educando con los libros, a través de la construcción del rincón de la lectura en el salón de clase.

- Taller 4: "El cuento un mundo para soñar”. Propósito: Comprender el texto "El Lápiz Mágico" y su incidencia en el desarrollo de la habilidad lectora.

- Taller 5: "El cuento, un mundo de vivencias creadoras y formadoras”. Propósito: Reconocer en el cuento "Boris un nuevo compañero en la escuela” valores aplicados a la vida cotidiana.

- Taller 6: "Explorando mi futuro". Propósito: reflexionar acerca de las profesiones, para Incorporarlo en su proyecto de vida, utilizando las experiencias de cuento "El gato Bombero".

- Taller 7: "Las diferencias que encantan”. Propósito: Valorar las diferencias como formas de enriquecimiento colectivo utilizando el cuento "Niña Bonita" 
En los últimos cuatro talleres se realizó actividades que incentivaron el uso y conocimiento de la lengua como: actividades de expresión oral, comprensión, aprendizaje de nuevo vocabulario y utilización del diálogo como medio de intercambio comunicativo en los cuales se favoreció la enseñanza-aprendizaje de la lectura.
Al final de cada uno de estos talleres se aplicó una guía evaluativa sobre comprensión lectora, teniendo en cuenta los componentes sintáctico, semántico y pragmático.

\section{Técnicas e Instrumentos para Recolección de la Información. (Véase tabla 1)}

Tabla 1: Técnicas e Instrumentos para Recolección de la Información.

\begin{tabular}{|c|c|c|}
\hline Fases & Técnica & Instrumento \\
\hline \multirow[t]{5}{*}{ Planificación } & $\begin{array}{l}\text { Revisión documental del PEI, mallas curriculares, } \\
\text { estándares, DBA, competencias, desempeños, resultados } \\
\text { obtenidos en el ISCE y prueba SABER } 2015 \text { y 2016, en } \\
\text { relación al área de lenguaje, y el Plan de Mejoramiento. } \\
\text { Observación- diagnóstico de prácticas pedagógicas } \\
\text { y cuadernos de niños de grado primero de los años } \\
\text { anteriores. }\end{array}$ & Diario de Campo. \\
\hline & Clase Entrevista & \\
\hline & Antecedentes y autores. & $\begin{array}{l}\text { Tareas: } \\
\text { Lectura del propio nombre. } \\
\text { Lectura de un texto. } \\
\text { Lectura de las cuatro palabras y una frase. } \\
\text { Nombres de las letras. } \\
\text { Asociación de letra sonido. } \\
\text { Unidades lingüísticas. }\end{array}$ \\
\hline & & Diario de campo. \\
\hline & & Fichas bibliográficas. \\
\hline Acción & $\begin{array}{l}\text { Propuesta "El Cuento Multimodal como Herramienta } \\
\text { para la Enseñanza- Aprendizaje de la Lectura”. }\end{array}$ & $\begin{array}{l}\text { Talleres } \\
\text { Diario de campo }\end{array}$ \\
\hline Observación & Observación & $\begin{array}{l}\text { Diario de campo } \\
\text { Resultados Clase entrevista } \\
\text { Guía competencia lectora }\end{array}$ \\
\hline Reflexión & Triangulación & Diario de campo \\
\hline
\end{tabular}

Fuente: construcción de las autoras. 


\section{Resultados y discusión}

Diagnóstico. Se evidenció que los docentes utilizan, en su mayoría, los métodos sintético y analítico en la iniciación alfabética porque, según ellos, ha dado resultados. Se valen de planas y silabas para formar palabras y frases, sin tener en cuenta los intereses, conocimientos previos, el proceso evolutivo, cognitivo y, lo más importante la esencia se la lectura, como lo es la construcción de significado y sentido. Producto de ello no se logra un acercamiento eficiente del niño en los procesos iniciales de la lectura ni se enseña esta con un propósito comunicativo. Al respecto, Maqueo (2015), expresa que se requiere que en la escuela se promueva un conjunto de habilidades lingüísticas, pragmáticas y sociales, en las cuales se suscite el gusto por la lectura. Así mismo se utiliza cartillas como Nacho y Coquito y se observa la preocupación porque los niños reconozcan la segmentación de las palabras sin atender a su significado global.

Al observar los cuadernos diligenciados por los estudiantes de grado primero en años anteriores, se observa la utilización de planas y el silabeo en el proceso de enseñanza de la lectura y la escritura, los cuales, según Hurtado et al (2003), "las reduce a acciones meramente perceptivas y motrices, donde lo importante consiste en dibujar y sonorizar bien las letras, es decir, se privilegia la mecanización sobre la comprensión" (p. 1), acciones que se pretenden abolir con la aplicación de la estrategia planteada.
Aplicación de la Clase Entrevista que según Grossi (2010) citado por Grupo CoNprende (2010) la cual "es un encuentro individual del docente con cada uno de los estudiantes convirtiéndose en un momento de aprendizaje para los dos” (p. 38), esta permite ubicar al estudiantes en el nivel de psicogénesis (presilábico 1, presilábico2, silábico, alfabético y alfabetizado) en su proceso lector, lo cual es de gran importancia pues tiene en cuenta el proceso de aprendizaje tanto evolutivo como cognitivo. La aplicación de la Clase Entrevista, arrojó que el 70\% de los niños se encuentran en nivel silábico de lectura, el 20 en nivel presilaabico 1 y el $10 \%$ en nivel presilabico 2 . El nivel silábico corresponde a la "fonetización", donde generalmente se utiliza una señal gráfica para cada unidad sonora (Viegas, 2012); por consiguiente, es necesario promover experiencias enriquecedoras que permitan la confrontación de los diferentes niveles de conceptualización de los niños, dinamizando el proceso de desarrollo de la lectura que, según Hurtado et al (2003), "realimenta las diversas producciones lingüísticas de los niños, permitiéndoles tomar conciencia de cómo están leyendo, generando un proceso de autocorrección $y$ transformación lingüística y cognitiva" (p. 15).

Acción. En la implementación de los talleres, y concretamente en los tres primeros, orientados a la motivación hacia la lectura se encontró que los niños se familiarizaron con diferentes herramientas y espacios que favorecieron la motivación a la lectura. 
Se notó en los estudiantes el gusto e interés por interactuar con los libros, especialmente con cuentos que tenían a su disposición en el salón de clase, los cuales podían leer y luego compartir sus ideas con los compañeros. Lo anterior facilitó a los estudiantes evocar y comprender los cuentos ya que podían reconstruir los sucesos con mayor facilidad.

Como resultado del desarrollo de los cuatro últimos talleres se encontró que:

- Al proyectar los cuentos multimodales, los niños se sentían animados a participar, luego de las diferentes actividades realizadas se notó que ellos recordaban con facilidad los hechos, acontecimientos y personajes y, lograban obtener una reflexión acerca del mensaje que les dejaba para su vida. Así mismo, al hacerles preguntas relacionadas con el texto se mejoró la participación respondiendo de manera adecuada las preguntas que se les hacían.

- En el desarrollo de las actividades de inicio de cada taller, en las cuales se ejercitaba la escucha activa, se observó el fortalecimiento de esta habilidad, mejorando así la competencia comunicativa. A demás se notó mayor fluidez y riqueza expresiva ya que los estudiantes mostraban menos timidez al hablar.

- En el desarrollo de las guías de competencia lectora, se evidenció una mejoría en el componente semántico, sintáctico y pragmático, lo cual facilitó a los estudiantes la comprensión de los cuentos trabajados, recuperando información explicita, identificado la estructura y reconociendo información sobre los propósitos del texto.

Para concluir este apartado es pertinente expresar que se volvió a emplear la Clase Entrevista la cual mostró los avances de los estudiantes en su nivel de psicogénesis de lectura al pasar de nivel silábico a nivel alfabético en el cual el niño comprende que las palabras son compuestas y obtiene la capacidad de articular los conocimientos construidos en cada uno de los ejes que componen el campo conceptual de la lectura y obtiene la capacidad de leer un texto sencillo que aborda asuntos de su realidad con comprensión (Viegas, 2012).

Se notó en los estudiantes el gusto e interés por interactuar con los libros, especialmente con cuentos que tenían en el salón de clase y los cuales se les facilitaban para leerlos $\mathrm{y}$, luego compartir sus ideas con los compañeros. A los estudiantes se les facilitó evocar y comprender los cuentos ya que podían reconstruir los sucesos con mayor facilidad al proyectar las imágenes sin el texto.

\section{Conclusiones}

Sin una sólida fundamentación teórica en relación con los proceso lectoescriturales es casi imposible desarrollar practicas pedagógicas eficientes para potenciar esta competencia en los educandos.

La clase entrevista es un instrumento que aporta al docente valiosa información para conocer el nivel de psicogenesis 
en que se encuentra el niño y de ahí propiciar actividades que estimulen el proceso evolutivo del niño.

El cuento multimodal es una excelente herramienta para fomenta el interés y el gusto por leer, ya que al manejar audio y video se estimula el desarrollo de las habilidades lectoras en el caso particular, de los niños.
El propiciar ambientes agradables para la lectura despierta la imaginación y creatividad en los niño, aspectos que favorecen el desarrollo de esta competencia.

Al utilizar una estrategia pedagógicodidáctica en la enseñanza-aprendizaje de la lectura mejora el desempeño escolar y la interacción maestroestudiante.

\section{Referencias Bibliográficas}

Acevedo, A. (2014). ¿Cómo funciona el cerebro de los niños? Bogotá, Colombia.: Grijalbo.

Alvarado, L. M. (2008). Características má relevantes del paradigma socio-critico: su aplicación en investigaciones. Revista Universitaria de Investigación, 193.

Bravo, V. L. (2002). La conciencia fonológica como zona de desarrollo próximo para el aprendizaje inicial de la lectura. Santiago, Chile : Pontificia Universidad Católica de Chile.

Carr, W., \& Kemmis, S. (1988). Teoría critica de la enseñanza. Barcelona, España.: Martínez Roca.

Colella, L. (2016). El problema filosófico y el sujeto de la enseñanza de la filosofía. Aportes desde las nociones de "identidad" y "universalismo" de Alain Badiou. Cuestiones De Filosofía, (16), 213-226. https://doi.org/10.19053/01235095.2401

Diaz, F., \& Hernández, G. (2010). Estrategias docentes para un aprendizaje significativo, una interpretación constructivista. México: MC Graw Hill.

Ferreiro, E., \& Teberosky, A. (1979). Los sistemas de escritura en el desarrollo del niño. México: Siglo XXI.

Feuerstein, R. (2011). Conciencia Educativa. Obtenido de Conciencia Educativa: http://concienciaeducativalatorred.blogspot.com.co/2011/11/reuvenfeuerstein-teoria-de-la.html

Freinet, C. (1981). El Método Natural de Lectura . Barcelona, España: Laia Barcelona.

Goodman, K. (2003). El aprendizaje y la lectura y la enseñanza de le lectura y la escritura. Enunciación, 77-98. Obtenido de Revista Enunciación Universidad 
Distrital: http://revistas.udistrital.edu.co/ojs/index.php/enunc/article/ view/2480/3463

Grupo Conprende. (2010). Apoyo didáctico para alfabetizadores. Bogotá, D.C. Colombia: CoNprende.

Guillen, J. C. (18 de Septiembre de 2014). Escuela con Cerebro. Obtenido de Escuela con cerebro: https://escuelaconcerebro.wordpress.com/2014/09/18/lamotivacion-escolar-siete-etapas-clave/

Gurdian, F. A. (2007). El paradigma cualitativo en la investigación socio-educativa. San José de Costa Rica: Agencia Española de Cooperación Internacional.

Hurtado, V. R., Serna, h. D., \& Sierra, J. L. (2003). Lectura y Escritura en la Infancia: Estratégias Pedagógicas para Facilitar su Construcción. Copacabana, Colombia: Fundación Bancolombia.

Jaramillo, A. A., \& Negret, P. J. (1991). La construcción de la lengua escrita en el grado cero. Bogotá D.C, Colombia: MEN.

Jiménez, M., \& Gordo, A. (2014). El cuento infantil: facilitador de pensamiento desde una experiencia pedagógica. Praxis \& Saber, 5(10), 151 - 170. https:// doi.org/10.19053/22160159.3027

Lara, S. N. (2008). Estrategias para la enseñanza aprendizaje de la lecto-escritura en primer grado de educación primaria. México: Universidad Tangamanga.

Latorre, A. (2008). La investigación-acción conocer y cambiar la practicaeducativa. Barcelona, España: Grao.

Lorenzo, J. R. (2012). Principio Alfabético y Aprendizaje de la Lectura. Argentina: Universidad Nacional de Cordova.

Maqueo, A. (2005). Lengua, aprendizaje y enseñanza. México: Limusa.

MEN. (1996). Lineamientos curriculares, lengua castellana. Bogotá: Magisterio.

MEN. (2007). Lectura y escritura con sentido y significado. Al tablero.

MEN. (2016). ¿Qué dice aquí?.. ¿Cómo se escribe esta palabra? Orientaciones para promover la lectura y la escritura emergente en el grado de transición, 47.

Mendoza, A. (2006). Didáctica de la lengua y la literatura. Madrid: Prentice Hall.

Montealegre, R., \& Forero, L. A. (2006). Desarrollo de la Lectoescritura, Adquisisción y Dominio. Acta Colombiana de Psicología, 25-40. 
Moya, G. A., \& Pinar, S. M. (2007). La interacción texto/ imagen en el cuento ilustrado. Un análisis multimodal. OCNOS $n^{-0} 3,21-38$.

Navarro, B. A. (2015). Beneficios de la lectura expresiva en la competencia lectora. Sevilla, España: Universidad de Sevilla.

Prieto, M. H., Rodríguez, K. A., \& Trujillo, V. M. (2015). Aprendiendo y jugando fortalecemos la comprensión lectora. Villavicencio, Colombia: Corporación Universitaria Minuto de Dios.

Rincón, B. G., Bustamante, G., \& Pérez, d. R. (1999). La enseñanza de la Lengua Escrita y de la Lectura. Colombia: Arango Editores.

Viegas, P. J. (2012). Didáctica socio interaccionista para docentes. Bogotá. 